\title{
Design, analysis and testing of the Optical Tube Assemblies for the ESO VLT Four Laser Guide Star Facility
}

\author{
R. Henselmans, ${ }^{*}$, , D. Nijkerk ${ }^{\mathrm{a}}$, M. Lemmen ${ }^{\mathrm{a}}$, N.Rijnveld ${ }^{\mathrm{a}}$, F.Kamphues ${ }^{\mathrm{a}}$ \\ ${ }^{\mathrm{a}} \mathrm{TNO}$, Stieltjesweg 1, $2628 \mathrm{CK}$, Delft, The Netherlands
}

\begin{abstract}
TNO has developed the Optical Tube Assemblies (OTAs) for the ESO VLT Four Laser Guide Star Facility. The OTAs are Galilean 20x beam expanders, expanding a $\varnothing 15 \mathrm{~mm}$ input beam $(25 \mathrm{~W}, 589 \mathrm{~nm} \mathrm{CW})$ to a steerable $\varnothing 300 \mathrm{~mm}$ output beam. TNO has recently successfully completed acceptance testing of the four units, showing compliance to the challenging requirements on output wavefront quality, thermally induced defocus under operational conditions, absolute pointing accuracy and polarization extinction ratio (PER).

TNO applied its corrective polishing in combination with the NANOMEFOS measurement machine to produce the $\varnothing 380 \mathrm{~mm}$ aspherical output lens, resulting in $20 \mathrm{~nm}$ rms output wavefront quality. The thermal behaviour of the system has been analyzed by combining optical, lumped mass and FE analyses. A design that is passively athermalized over a large temperature range as well as under the influence of thermal gradients has been developed. Extensive thermal testing has shown a thermally induced defocus of less than 0.15 waves under the operational conditions of $0-15^{\circ} \mathrm{C}$ and upto $-0.7^{\circ} \mathrm{C} / \mathrm{hr}$ gradient. A custom tip-tilt mechanism was designed to steer the output beam over a 4.8 arcmin radius, with less than 0.1 " $(3 \sigma)$ accuracy at $1 \mathrm{~Hz}$ update rate. The PER was also measured under operational (thermal and tilt) conditions and demonstrated to be well above $99 \%$. This paper describes the design, modelling and analysis, and the test results of these instruments.
\end{abstract}

Keywords: Optical Tube Assembly, OTA, beam expander, athermal design, high-power laser guide star

\section{THE ESO VLT 4LGSF}

ESO (the European Southern Observatory) is equipping the Unit Telescope Yepun (UT4), part of the Very Large Telescope (VLT) located in the Atacama Desert in Chile, with an adaptive optics facility (AOF) [1]. This system will correct for atmospheric turbulence to improve the telescope's performance. To determine the wavefront distortion, a bright (natural) star nearby the faint object under study is normally used as a reference, but this approach limits sky coverage to about $1 \%$. To overcome this problem, an artificial Laser Guide Star (LGS) can be created, by exciting Sodium atoms in the atmosphere between 90 and $100 \mathrm{~km}$ altitude with a powerful $589 \mathrm{~nm}$ laser. ESO has developed a concept for a compact laser guide star unit for use in future Adaptive Optics (AO) systems. A small powerful laser is combined with a telescope that launches the beam, creating a single modular unit that can be mounted directly onto a large telescope. Four of these compact laser guide stars will be used for the new VLT Four Laser Guide Star Facility (4LGSF) (Figure 1). The technology will also serve as a testbed ahead of the construction of the future European Extremely Large Telescope, which will also have multiple laser guide star units.

\footnotetext{
* Corresponding author: rens.henselmans@tno.nl
} 
The 4 LGSF consists of a high power 25 W CW $589 \mathrm{~nm}$ laser, a Beam Conditioning and Diagnostics System (BCDS) and an Optical Tube Assembly (OTA). TNO has developed the OTA laser launch telescopes and successfully demonstrated its performance under operational conditions.

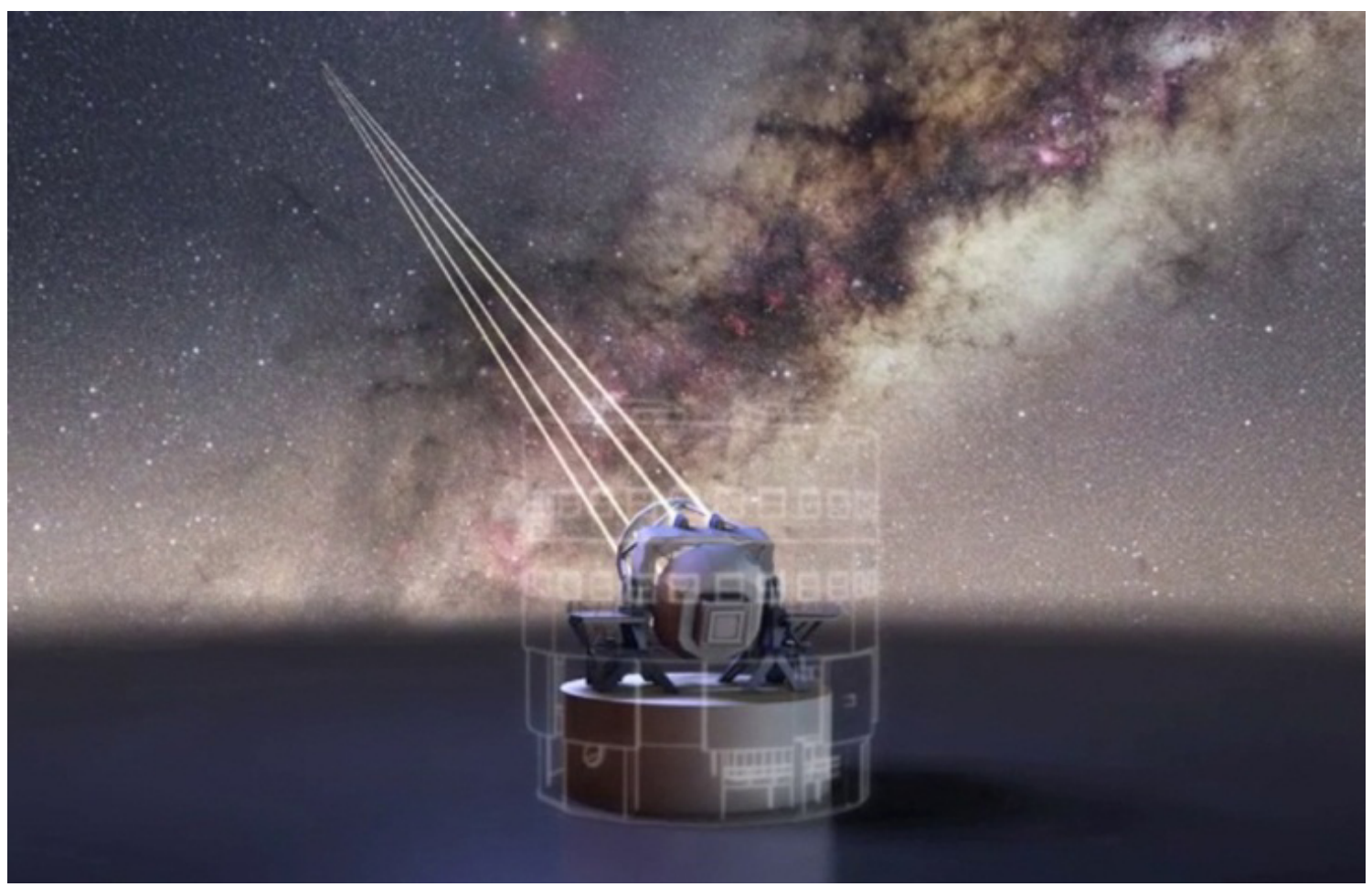

Figure 1: Artist impression of the VLT Four Laser Guide Star AO Facility (CESO)

\section{OTA DESIGN}

The OTAs are 20x Galilean beam expanders, consisting of an entrance lens L1, a steerable $45^{\circ}$ mirror (Field Selector Mirror, FSM) and a large aspherical exit lens L2 (Figure 2). They expand a $\varnothing 15 \mathrm{~mm}$ input beam to a $\varnothing 300 \mathrm{~mm}$ output beam, and operate at $589 \mathrm{~nm}$ with $25 \mathrm{~W}$ of continuous laser power. The main requirements are shown in Table 1, together with the achieved results.

\begin{tabular}{|l|l|l|}
\hline Requirement & Value & Achieved \\
\hline Transmitted wavefront error (excl focus and tilt) & $<50 \mathrm{~nm} \mathrm{rms}$ & $17-23 \mathrm{~nm} \mathrm{rms}$ \\
\hline Thermally induced defocus & $<0.2$ waves PV & $\sim 0.15$ waves PV \\
\hline Pointing accuracy on-sky & $<0.3$ " $(3 \sigma)$ & $<0.2$ ' $(3 \sigma)$ \\
\hline Polarization extinction ratio (PER) & $>98 \%$ & $>99.5 \%$ \\
\hline Throughput & $>95 \%$ & $97.7 \%$ \\
\hline
\end{tabular}

Table 1: OTA main requirements and achieved results 
The OTA must achieve this performance under the operational conditions. The operational temperature is $0-15^{\circ} \mathrm{C}$ with $10^{\circ} \mathrm{C}$ average, with a maximum gradient of $-0.7^{\circ} \mathrm{C} / \mathrm{hr}$ for 8 hours. The OTA design is passively athermalized over a large temperature range as well as under the influence of thermal gradients [2]. The pointing can vary between $0-60^{\circ}$ Zenith angle. The air pressure varies between the locations: 1013 mbar in Delft (TNO), 960 mbar in Garching (ESO) and 750 mbar in Paranal (VLT). Extensive testing was done to verify the performance under the operational conditions.
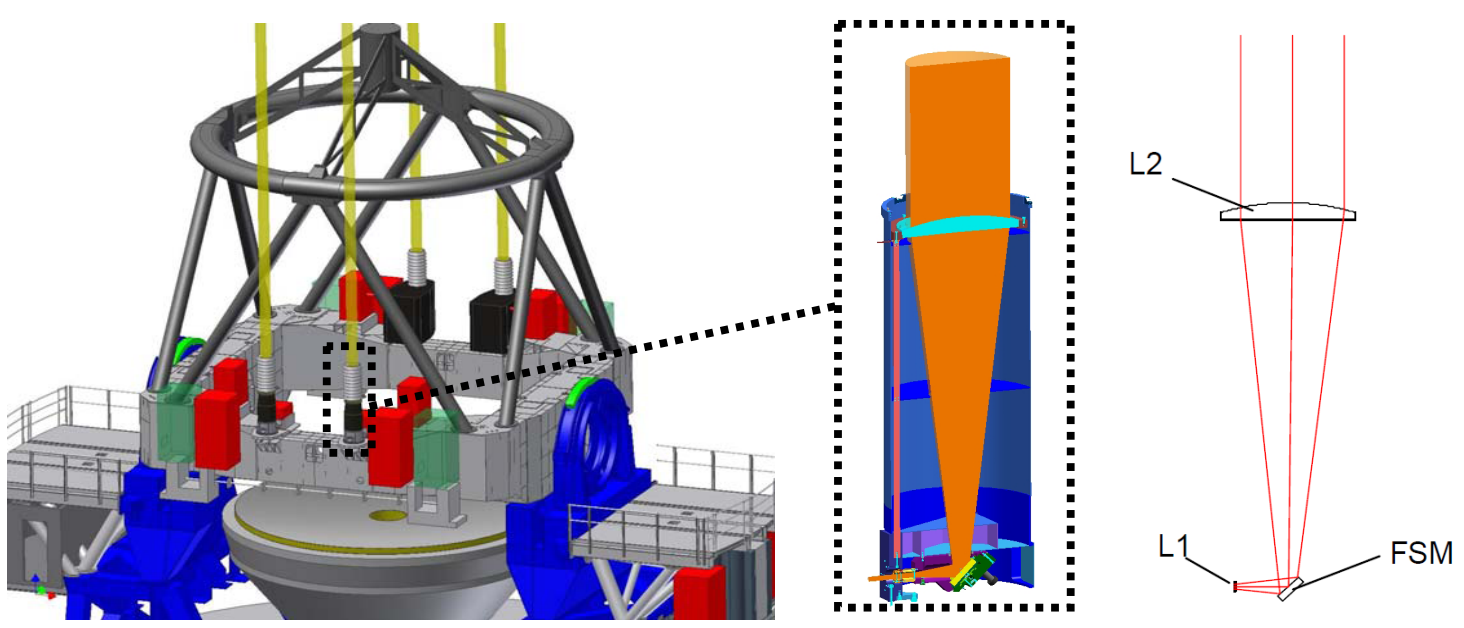

Figure 2: The OTA location on the telescope, and the optical design (CESO/TNO)

\section{OPTICAL DESIGN}

The optical design is shown in Figure 2. It consists of a $\varnothing 30 \mathrm{~mm}$ double concave entrance lens (Fused Silica), a $\varnothing 100$ $\mathrm{mm}$ multi-layer coated mirror (Zerodur) and a $\varnothing 380 \mathrm{~mm}$ conical aspherical exit lens (NBK7) with about $6.8 \mathrm{~m}$ concave radius in the front, and $0.64 \mathrm{~mm}$ convex radius an the back surface. This asphere is necessary to maintain the wavefront quality for the varying field of view. The OTA internal volume is sealed against dust, and contains filtered air at ambient pressure. The nominal wavefront quality of the design, including alignment and manufacturing tolerances is $34 \mathrm{~nm} \mathrm{rms}$ (note that $17-23 \mathrm{~nm}$ rms was achieved). The outer dimensions of the system are $\varnothing 450 \mathrm{~mm} \times 1.3 \mathrm{~m}$.

The defocus of the system is determined by four components: L1, L2, the air and the distance between L1 and L2. For the lenses this is caused by the $\mathrm{dn} / \mathrm{dT}$, the CTE, the bulk temperature and the temperature distribution. For the air this is a function of pressure, temperature and humidity. For the structure this depends on the CTE, the average temperature and the temperature distribution. The sensitivity of each component was determined using CodeV (Table 2). To make the system passively athermalized, the structure between L1 and L2 must have +0.04283 waves $/ \mathrm{K}$, which is equal to a CTE of $3.2 \mu \mathrm{m} / \mathrm{m} / \mathrm{K}$.

\begin{tabular}{|l|l|l|}
\hline Component & Sensitivity & Relative \\
\hline L1 & -0.01356 waves/K & $-20 \%$ \\
\hline L2 & -0.05731 waves $/ \mathrm{K}$ & $-80 \%$ \\
\hline Internal air & +0.02804 waves $/ \mathrm{K}$ & $+40 \%$ \\
\hline Structure & +0.04283 waves $/ \mathrm{K}$ & $+60 \%$ (Goal) \\
\hline
\end{tabular}

Table 2: Defocus sensitivities 


\section{ATHERMAL DESIGN}

Two concepts were evaluated (Figure 3). In the first, a carbon tube with a tuned CTE is used to determine the spacing between L1 and L2. This tube also constrains the L2 in lateral direction. This design is light and stiff, but has a relatively high uncertainty for the CTE. It has a low thermal time constant, making the tube (and the internal air) much faster than L2. The carbon design is expected to be relatively difficult and costly to manufacture.

The second concept creates the desired CTE by combining about $0.2 \mathrm{~m}$ stainless steel with $1 \mathrm{~m}$ Invar. The Invar is implemented as 3 rods constraining piston, tip and tilt of the L2. A steel tube constrains the L2 in lateral direction. This design has similar stiffness as the carbon design, but with higher mass. The uncertainty on the expansion coefficient is smaller, and the time constant is much larger. This slows down the internal air, keeping the defocus determining components better in pace. It is also expected to be easier and less costly to manufacture.

\section{Carbon tube with tuned $\alpha$}

- Carbon tube does 6 DOF of L2

- Tuned to $3.2 \mu \mathrm{m} / \mathrm{m} / \mathrm{K}$

- Light, stiff

- $\alpha$ uncertain $(\sim 0.5 \mu \mathrm{m} / \mathrm{m} / \mathrm{K})$

- Small thermal time constant

- Difficult to manufacture

- Higher cost (interfaces)

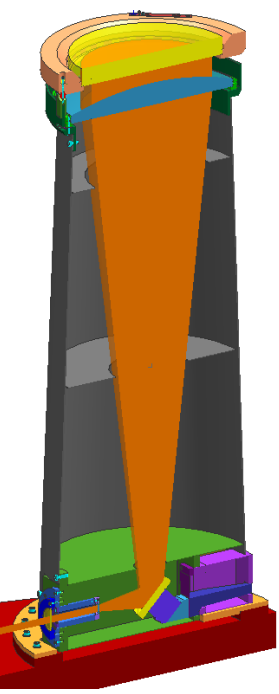

\section{Steel tube with Invar struts}

- 3 Invar struts do piston \& tilt of L2

- Steel tube does lateral DOFs

- $1 \mathrm{~m}$ Invar $+0.2 \mathrm{~m}$ steel $\approx 3.2 \mu \mathrm{m} / \mathrm{m} / \mathrm{K}$

- Function separation

- $\quad$ Larger mass

- $\quad \alpha$ less uncertain

- Larger thermal time constant

- $\quad$ Easier to manufacture

- Lower cost

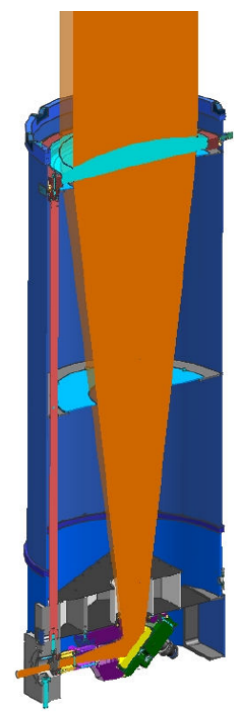

Figure 3: Concept trade-off

A lumped mass model was made of both concepts, with which the defocus performance was simulated. In the steel and Invar concept, the time-constants are better matched, resulting in less defocus. By making some components heavier than necessary from a strength point of view, the thermal matching was optimized. Figure 4 shows the final expected behavior (temperature difference from external temperature shown), with -0.013 waves defocus after 6 hours.
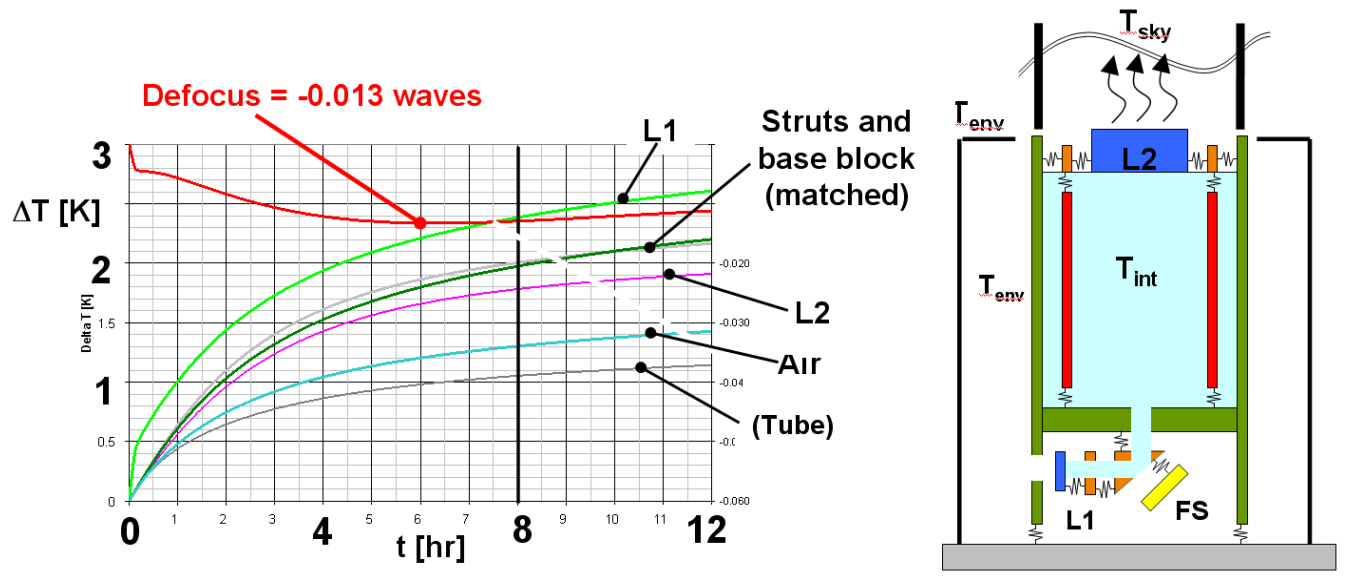

Figure 4: Lumped mass model defocus simulation

FEM analysis was also performed to calculate the gradients in the lenses. All combined, a nominal defocus of nearly zero was expected, with a model uncertainty of 0.122 waves PV. 


\section{FIELD SELECTOR MECHANISM}

The Field Selector Mechanism (FSM) design and testing is described in more detail in [3], a summary is given here. To achieve 4.8 arcmin radius field of view on-sky, the FSM has to tilt up to $\pm 6.1 \mathrm{mrad}$, in combination with less than 1.5 $\mu \mathrm{rad}$ RMS absolute accuracy. The maximum settling time for a 1 arcsec step on-sky is $0.2 \mathrm{sec}$. The FSM design (Figure 5) consists of a Zerodur mirror, bonded to a membrane spring and strut combination to allow only tip and tilt. Since the range is too large for piezos, two (self-locking) spindle drives actuate the mirror, using a stiffness based transmission to increase resolution. Absolute accuracy is achieved with two differential inductive sensor pairs. Friction in the spindle drive is overcome by creating a local velocity control loop between the spindle drives and the shaft encoders. Accuracy is achieved by using a cascaded low bandwidth control loop with feedback from the inductive sensors.

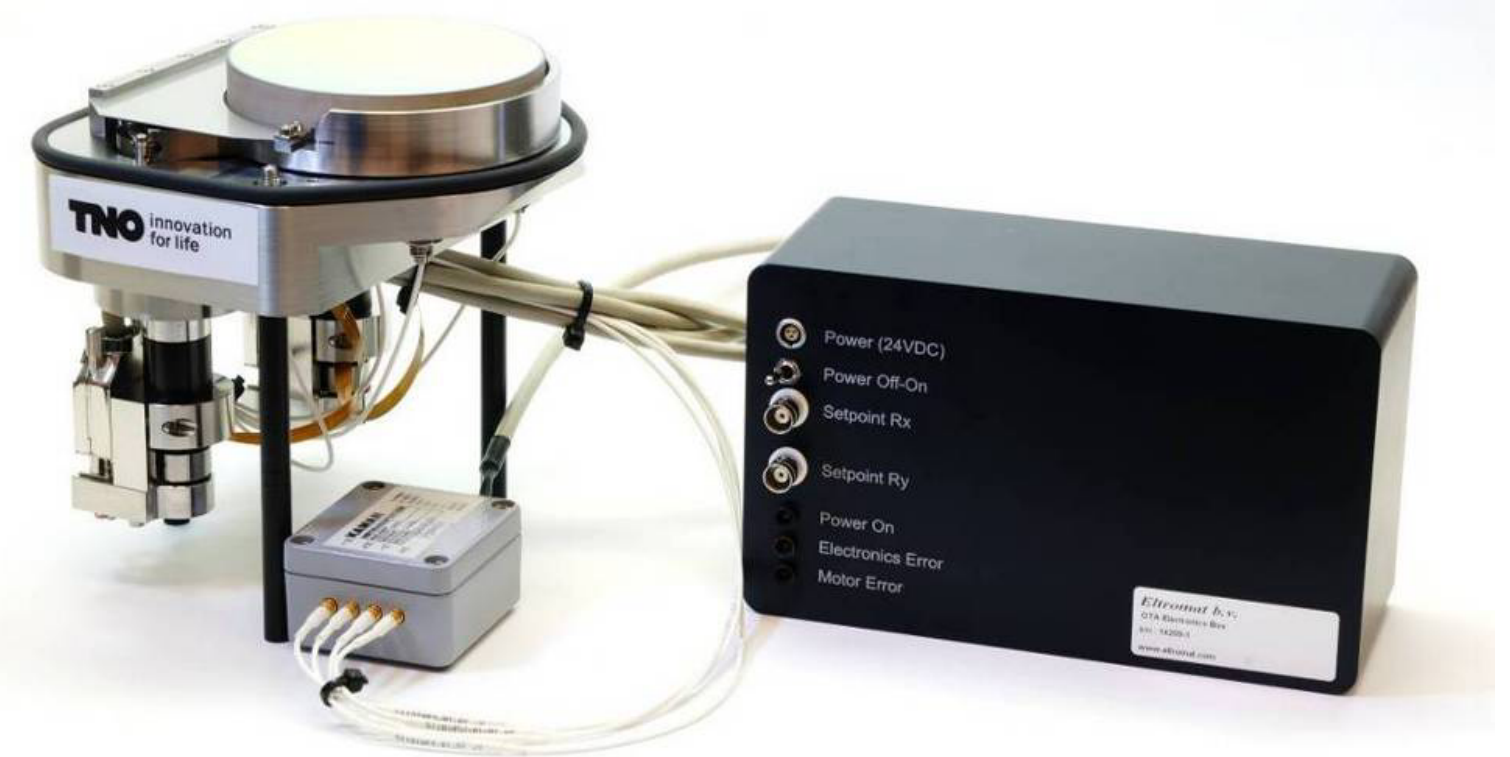

Figure 5: Field Selector Mechanism

The pointing jitter and settling time of the FSM are measured with an autocollimator. The amplitude spectral densities of the pointing jitter of the FSM mirror, show that the jitter of the mirror is only increased below the inductive sensor feedback loop, as can be expected. Integration of these spectra shows that the RMS of the pointing jitter down to $0.2 \mathrm{~Hz}$ is below $0.4 \mu \mathrm{rad} \mathrm{rms}$. An additional measurement is performed to show the behaviour of the pointing jitter towards lower frequencies, up to 0.07 " on-sky in worst case. All step responses are within 0.2 seconds. The FSM therefore meets its requirements.

\section{L2 FABRICATION}

The fabrication of L2 is described in more detail in [4], a summary is given here. The aspherical L2 lenses were manufactured using TNO's unique manufacturing facilities for aspherical and freeform optics. The process was hereto optimized for minimal mid-spatial content and low roughness $(1 \mathrm{~nm} \mathrm{Rq}$ achieved). The asphere was polished from $5 \mu \mathrm{m}$ $\mathrm{PV}$ to $24 \mathrm{~nm}$ rms in 6 runs. Figure 6 shows the final result as measured with the NANOMEFOS measurement machine [5]. This non-contact measurement machine typically obtains $2 \mathrm{k} \times 2 \mathrm{k}$ points in about 15 minutes, giving interferometric resolution with nm level accuracy (Figure 7). The resulting form error is about $24 \mathrm{~nm}$ rms, of which $14 \mathrm{~nm}$ rms is midspatial content. This contributes about $7 \mathrm{~nm}$ rms to the wavefront error. A custom hardened AR coating was applied with less than $0.2 \%$ reflectivity. 

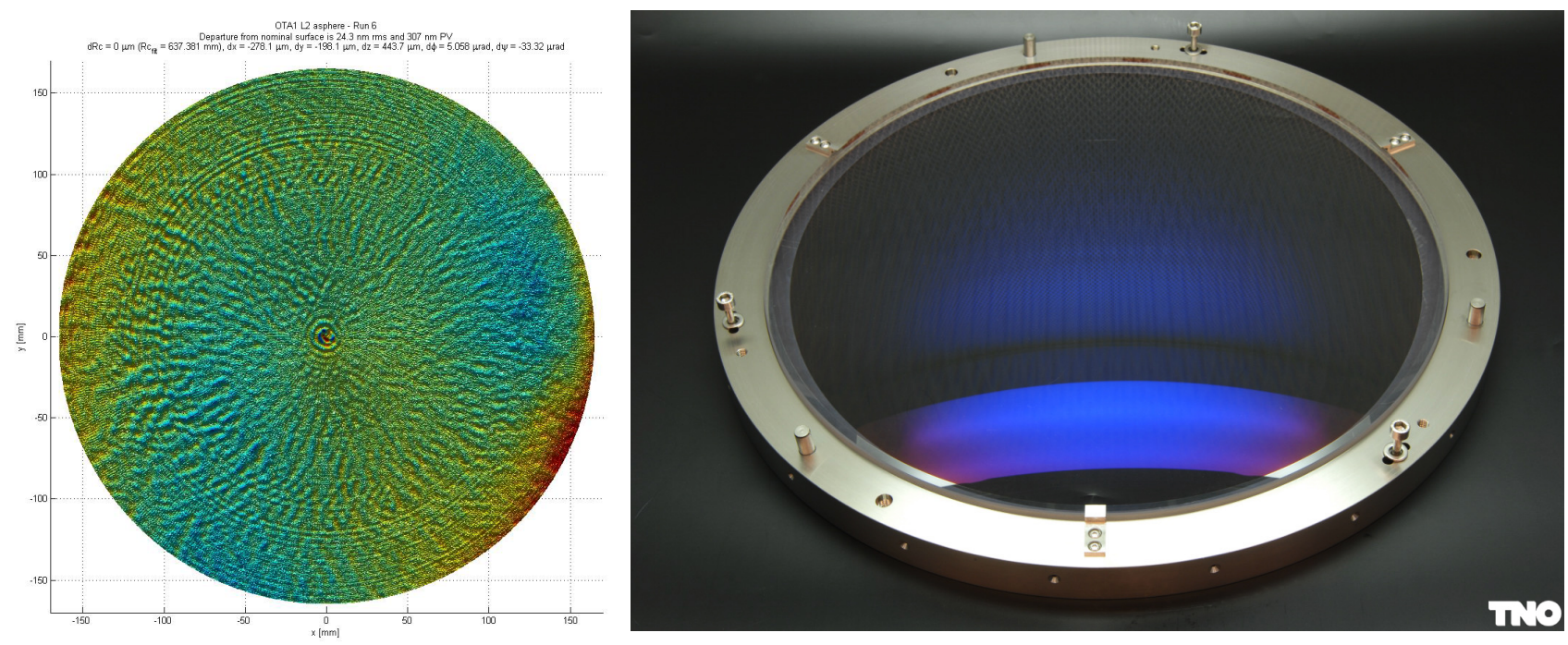

Figure 6: L2 asphere final result

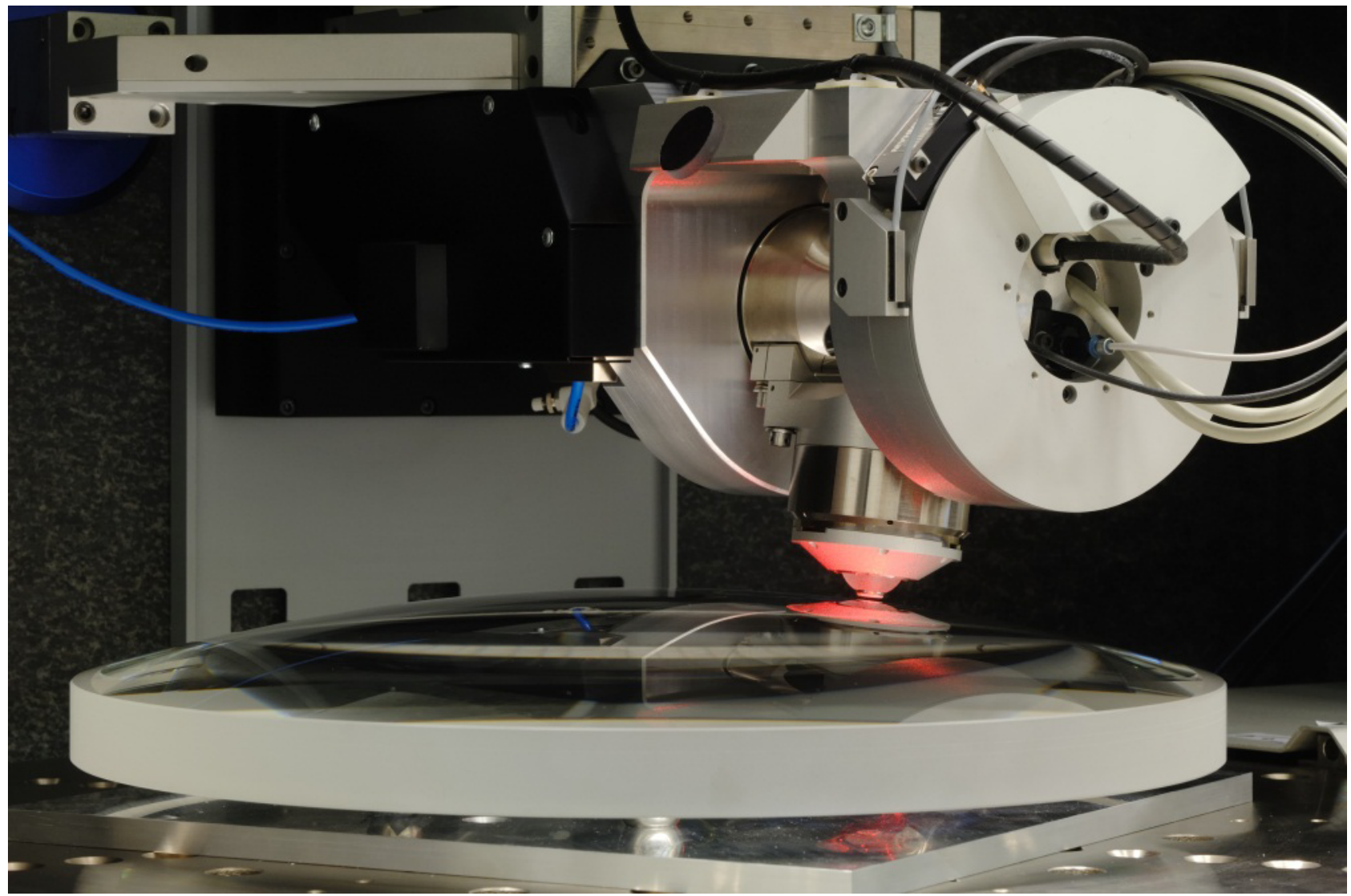

Figure 7: Non-contact measurement of the L2 convex asphere on NANOMEFOS

Proc. of SPIE Vol. $844784474 \mathrm{~N}-6$ 


\section{VERIFICATION TESTING}

\subsection{Wavefront error and defocus}

One of the completed OTAs is shown in Figure 8. The OTA wavefront quality was tested first by placing a reference flat mirror on top of the OTA and measuring the transmitted wavefront quality with a Trioptics $\mu$ Phase interferometer. The result is $17-23 \mathrm{~nm} \mathrm{rms}$, depending on the field of view (Figure 8, right). The correspondence with the L2 measurement can clearly be seen.
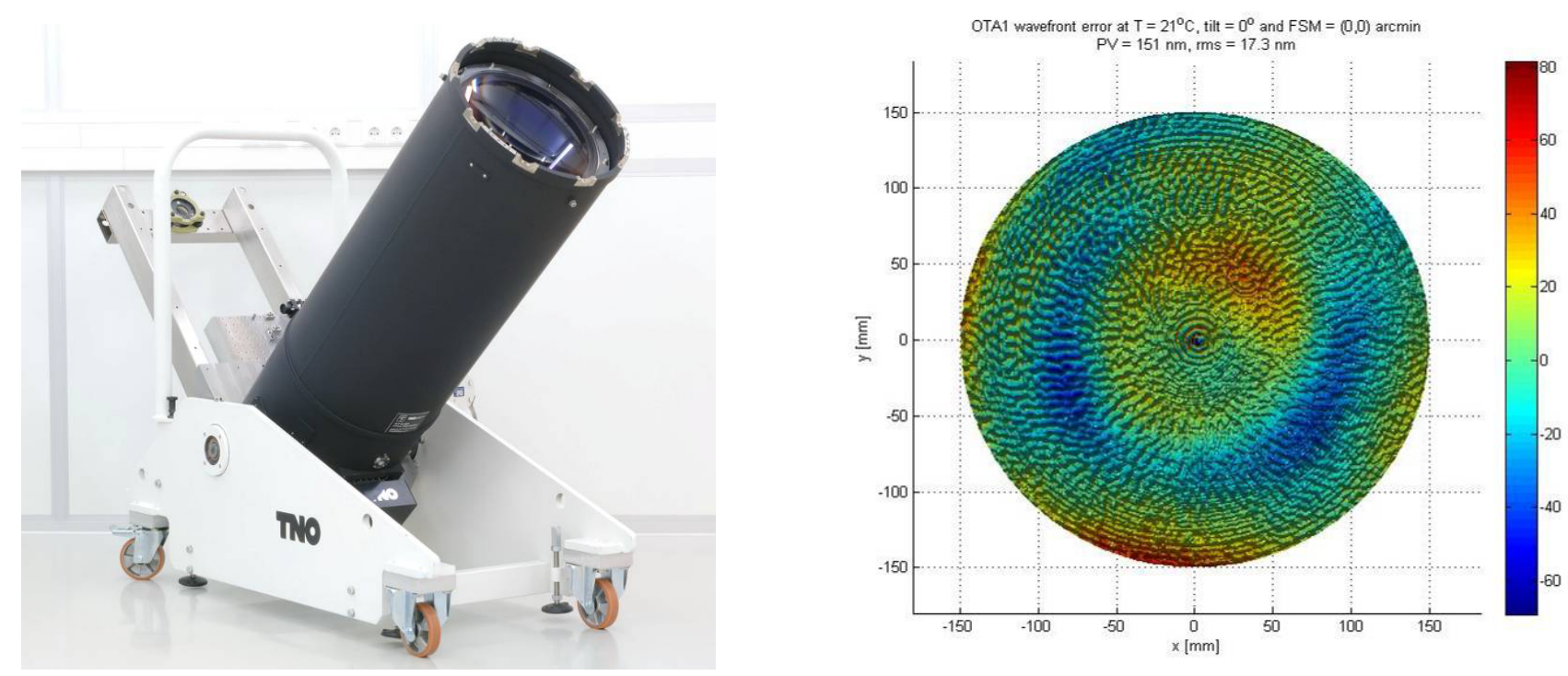

Figure 8: Completed OTA and wavefront measurement result

In order to get a feel for the WFE and defocus behavior under thermal transient conditions, a number of thermal tests were performed at TNO in an semi-controlled environment (not a climatic test chamber). The results were used to fine tune the performance model (pressure sensitivity was added, thanks to bad summer weather in the Netherlands). A complete and detailed understanding of the behavior of the OTA was obtained before shipping the instrument to ESO for controlled testing in their climatic chamber. A thermal model update was made after initial testing, based on detailed test results. This model now enables ESO to feedforward the defocus, to further improve the OTA defocus performance.

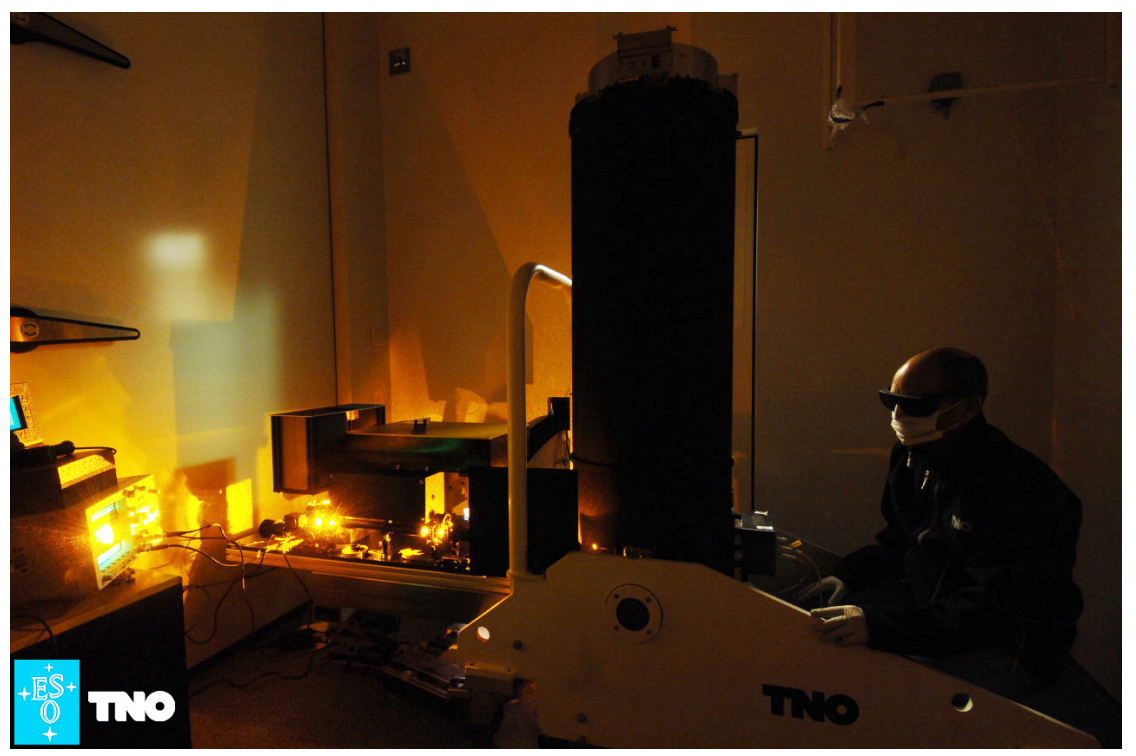

Figure 9: High-power thermal testing in ESO climate chamber 
Controlled thermal testing in combination with the high-power laser was performed in the ESO climate chamber (Figure 9). No surprises were found here, and the OTA meets its requirements for WFE and thermally induced defocus.

The measurement data of the long cooldown from $8^{\circ} \mathrm{C}$ to $1^{\circ} \mathrm{C}$ during 10 hours is shown in Figure 10 .The maximum defocus occurs after about 8 hours and amounts 0.19 waves.
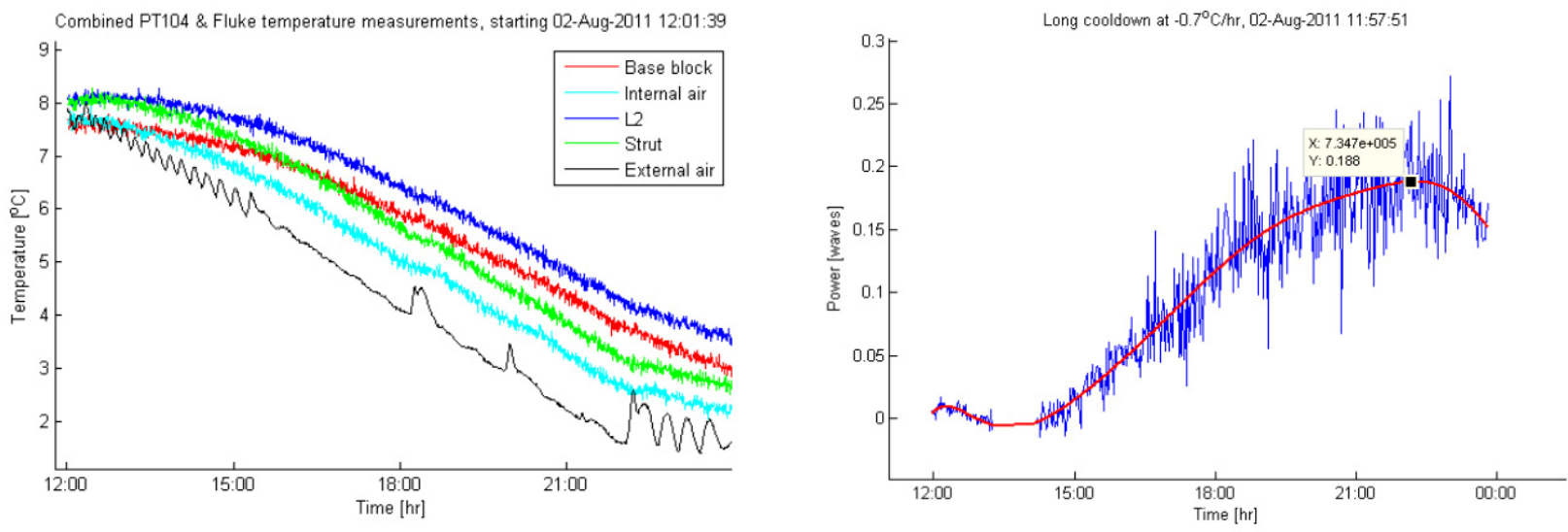

Figure 10: Defocus under thermal transient conditions

The defocus due to thermal gradients in L2 from laser absorption is no part of the thermally induced defocus budget. It has however been tested twice, to quantify the influence. Both tests show a similar defocus of about 40-60 nm or 0.070.1 wave PV.
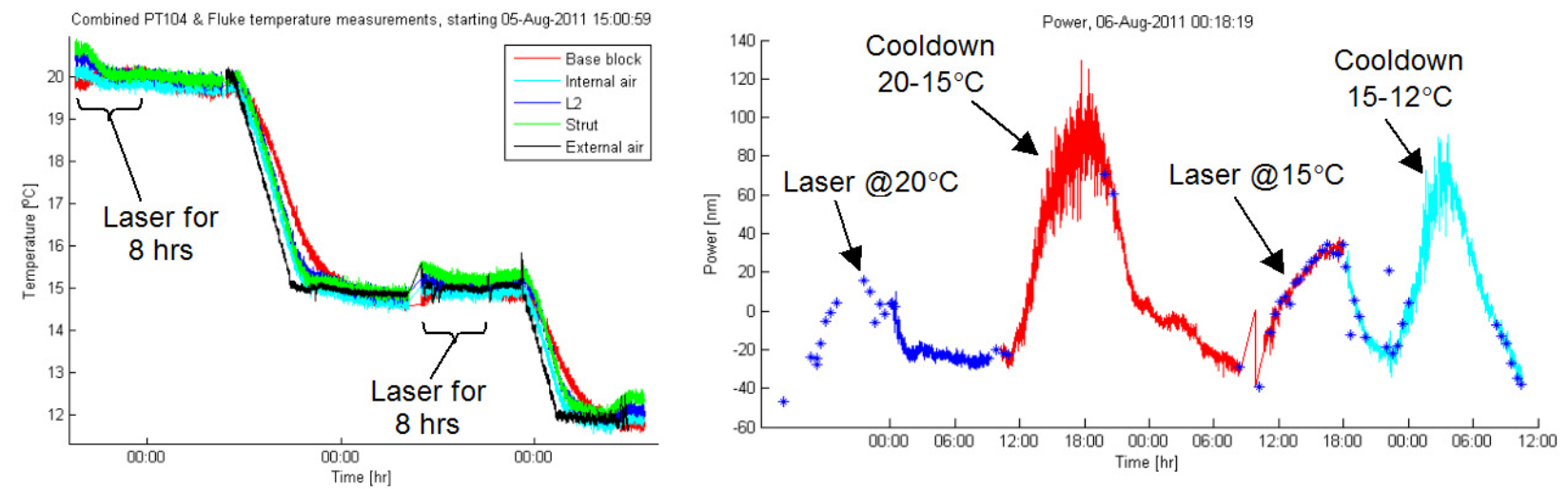

Figure 11: Defocus under transient and laser power conditions

\subsection{On-sky pointing accuracy}

The OTA pointing performance was verified with a mirror mounted onto 3 Invar struts, such that the mirror is always parallel to the baseplane (Figure 12). A triangular tube with a platespring constrains the mirror in lateral direction. The OTA pointing hysteresis as a function of tilt was determined by tilting it from $0^{\circ}$ to $60^{\circ}$ and back 5 times. 

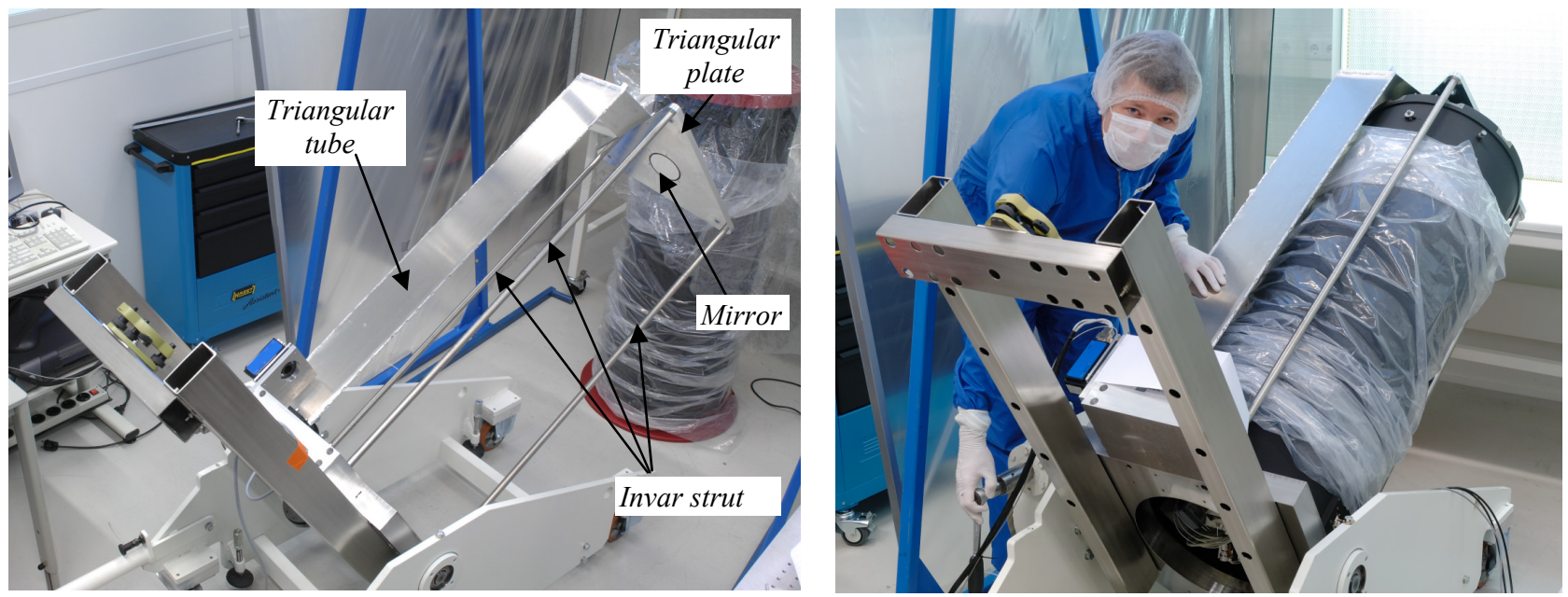

Figure 12: Pointing hysteresis measurements with Pointing Test Setup installed

Figure 13 shows the measurement results in arcseconds for OTA4. Note that in this test, the FSM was switched on, which introduces some measurement noise due to the phase stepping of the Trioptics $\mu$ Phase interferometer (which was used for the tilt measurements). Some drift of the test setup can also be seen, which is caused by thermal disturbances induced by the test engineer (this was verified with a number of additional tests). Separate tests also showed that the hysteresis of the Pointing Test Setup greatly contributed to the overall measured hysteresis, due to limited stiffness of the test trolley base plate. The UT centrepiece baseplate has a much higher stiffness and mechanical hysteresis is expected to be negligible. Taking this into account, the pointing hysteresis caused by tilting the OTA is within budget.
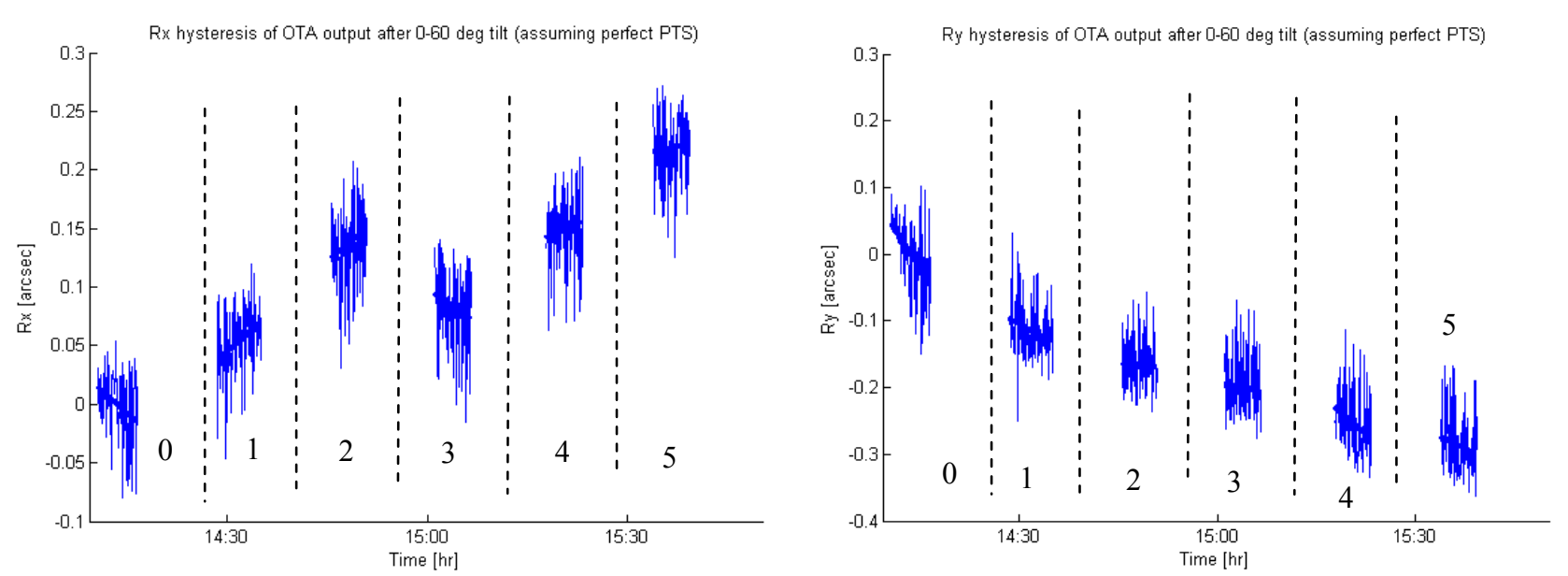

Figure 13: OTA pointing hysteresis in $\operatorname{arcsec}$ (OTA 4), test setup is main contributor to result

The absolute on-sky pointing accuracy of the full system was measured with the same setup. High-frequency measurements are not possible with the $\mu$ Phase interferometer, so this test only verifies the conversion of the direction and scaling in the electronics. Figure 14 shows the measurement results, again for OTA4. The $\mu$ Phase can handle up to about 40 fringes, which corresponds to 12" on-sky, a full range check is therefore not possible. A calibration table of each FSM was hereto generated separately before integration into the OTAs. 


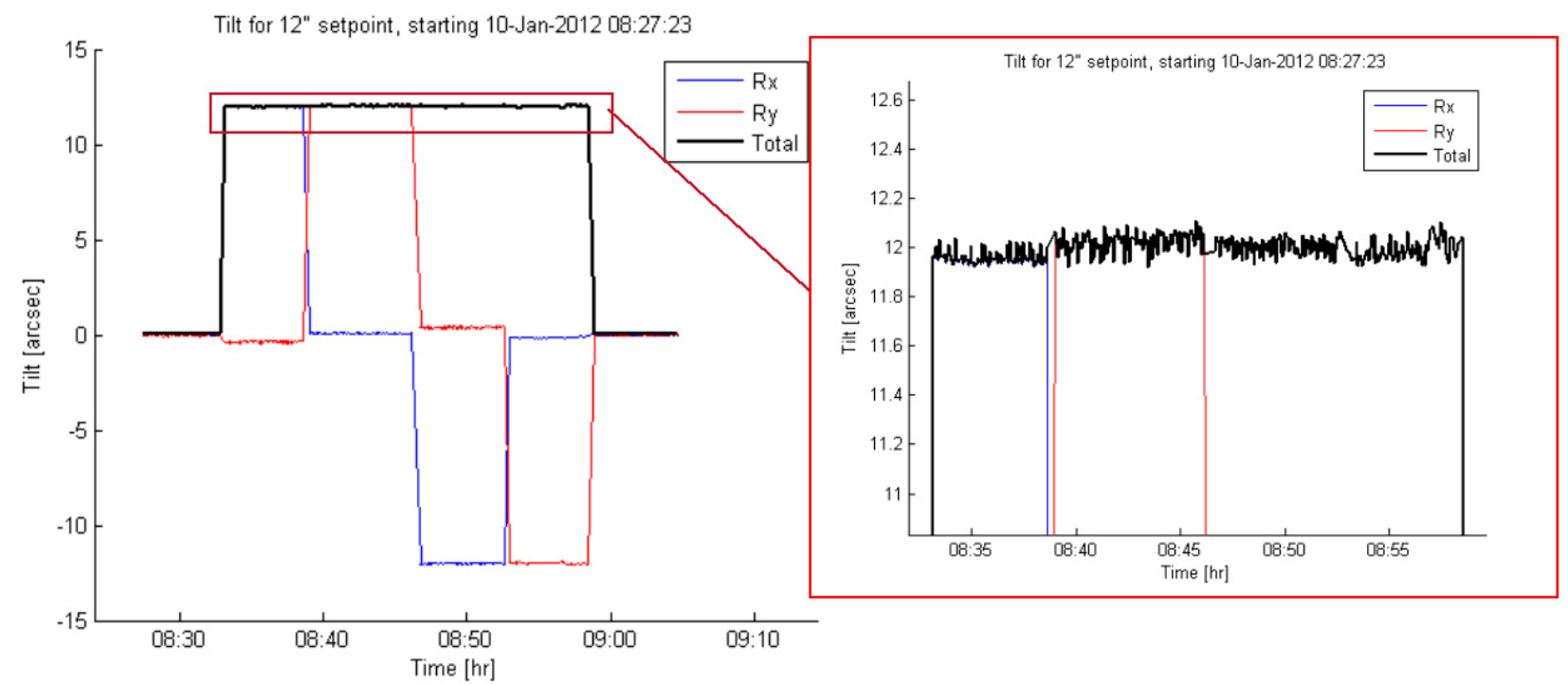

Figure 14: On-sky pointing verification (OTA 4)

The full system pointing hysteresis for the full range of the FSM was also tested in the same setup. The FSM was therefore moved from the $\left(0^{\prime}, 0^{\prime}\right)$ position to all extreme positions and back. The results are shown in Figure 15. Due to the (small) pointing jitter of the FSM, in combination with the phase stepping of the $\mu$ Phase, some noise is present. Some drift can also be seen. The hysteresis is however well within budget.

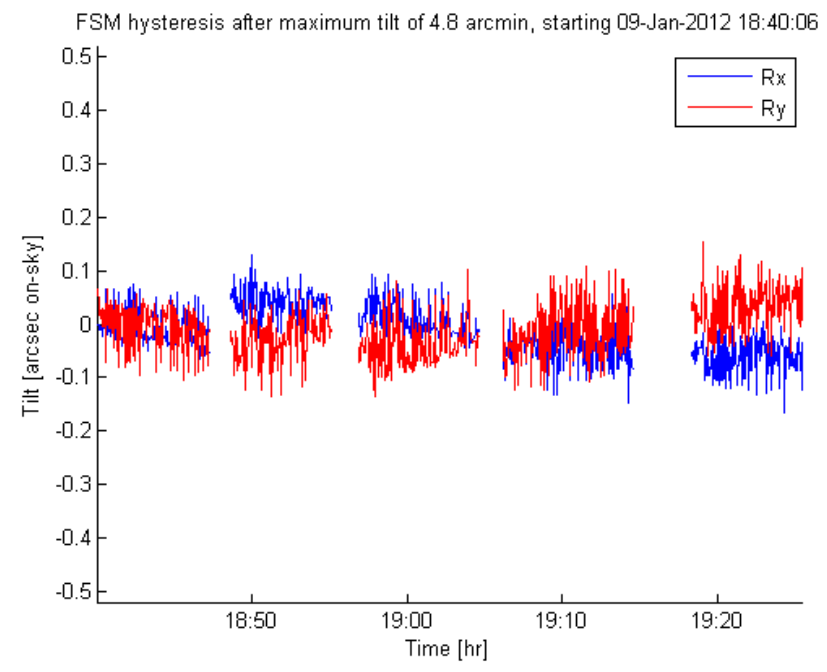

Figure 15: Full system pointing hysteresis of FSM (OTA 4)

In total, the measured non-repeatable random pointing accuracy of the OTA is 0.19 " $(3 \sigma)$ on-sky. The hysteresis of the pointing test setup itself is however the largest contributor to this value and as explained is expected to be much smaller, due to limited stiffness of the test setup and thermal instabilities caused by test operators. The 0.19 " is therefore a conservative number, but 0.07 " is expected based on analysis of the other contributing factors (FSM jitter, FSM hysteresis and FSM thermal zero-point drift). 


\subsection{Polarization Extinction Ratio (PER)}

A horizontally polarized input beam is converted to right-handed circularly polarized output. The Polarization Extinction Ratio (PER) was first tested at $20^{\circ} \mathrm{C}, 0^{\circ}$ tilt at low power $589 \mathrm{~nm}$ on OTA 1 . The result, weighted with the Gaussian intensity profile, is shown in Figure 16. The measurements shows a PER of 99.7\%, which is well above the required $98 \%$.
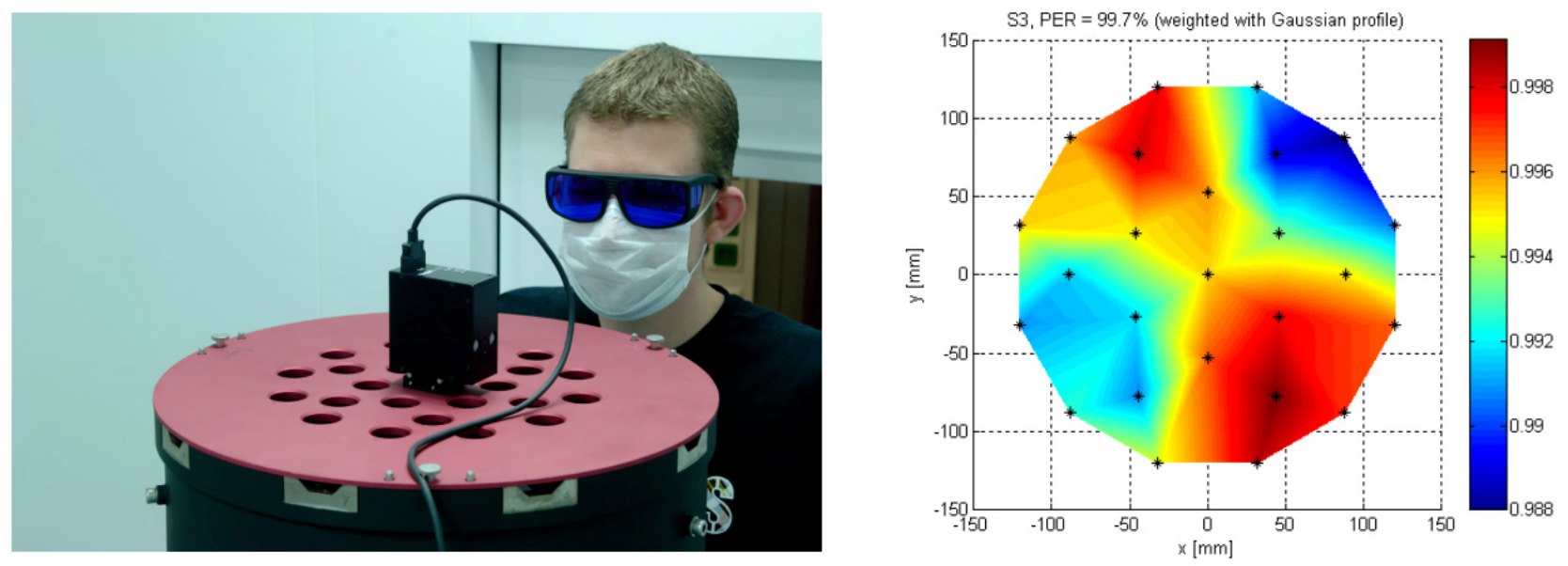

Figure 16: Low power PER of OTA 1 at T $=20^{\circ} \mathrm{C}$, tilt $=0^{\circ}, \lambda=589 \mathrm{~nm}$

Gravity induced stress inside the lenses may cause birefringence. The PER as function of tilt was measured on OTA 4 (Figure 17. The results are shown in Figure 18. The PER at $0^{\circ}$ is $99.6 \%$ and $99.5 \%$ at $60^{\circ}$. Also, the overall shape matches the $0^{\circ}$ tilt well. This confirms the PER is insensitive to the gravity direction.

High power laser PER measurements will be done by ESO at system level.

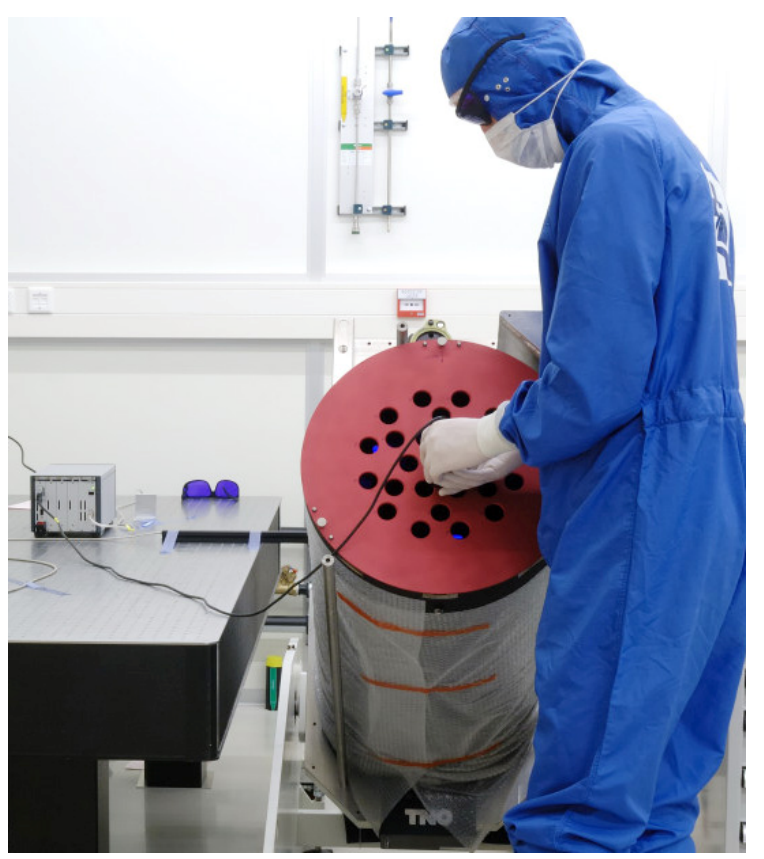

Figure 17: PER measurement on at $60^{\circ}$ tilt 

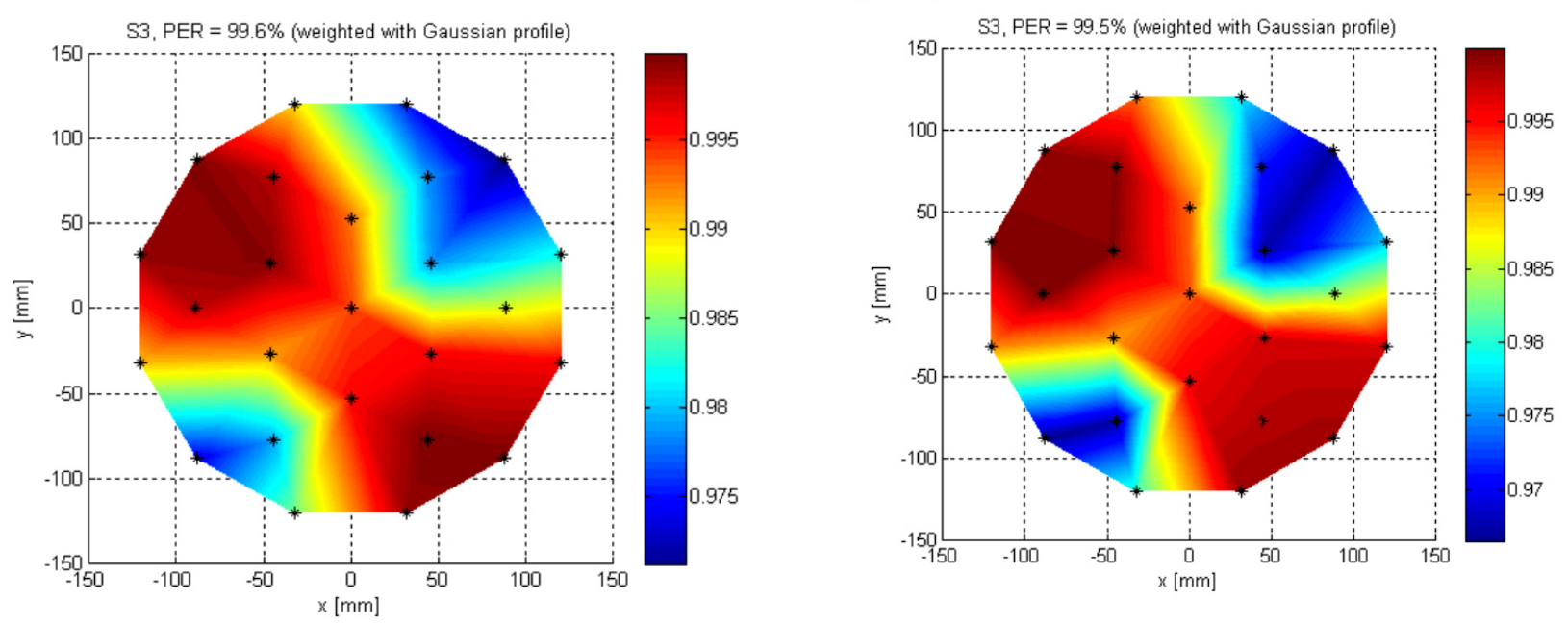

Figure 18: Low power PER of OTA 4 at $\mathrm{T}=20^{\circ} \mathrm{C}$, tilt $=0^{\circ}$ (left) and $60^{\circ}$ (right), $\lambda=589 \mathrm{~nm}$

\section{CONCLUSIONS AND FUTURE ACTIVITIES}

Design, analysis and testing of the OTAs was described. A passively athermalized design was made by optimizing the CTE and time-constants of the system's components. The design and realization of the other two main components, the FSM and L2, was also described. The design of the OTAs was described. A passively athermalized design was made by optimizing the CTE and time-constants of the system's components. The design and realization of the other two main components, the FSM and L2, was also described. The verification test programme was completed successfully and OTA meets all of its requirements. The achieved performance compared to the requirements is shown in Table 1.

TNO has delivered all four Optical Tube Assemblies to ESO and will support the system level integration where necessary. The OTA design can also be applied for other laser guide star facilities for 10 meter class telescopes or 30 meter class ELTs.

\section{ACKNOWLEDGEMENTS}

TNO would like to thank ESO for awarding the contract for the realization of the Optical Tube Assemblies for the Very Large Telescope, and for making their climate chamber and assistance available for testing of the OTAs.

All photographs were provided by Fred Kamphues.

\section{REFERENCES}

[1] www.eso.org/sci/facilities/develop/ao/images/AOF_Booklet.pdf

[2] R. Henselmans, D. Nijkerk, M. Lemmen, F.Kamphues, "Athermal design of the Optical Tube Assemblies for the ESO VLT Four Laser Guidestar Facility”, SPIE Integrated modeling Symposium, 2011

[3] Rijnveld, N., R. Henselmans, B. Nijland, "A tip/tilt mirror with large dynamic range for the ESO VLT Four Laser Guide Star Facility", SPIE Optics \& Photonics, 2011

[4] Gubbels, G., R. Henselmans, C. van Drunen, "Flexible manufacturing of large aspheres for VLT's Optical Tube Assemblies", SPIE Optics \& Photonics, 2011

[5] Henselmans, R., "Non-contact Measurement Machine for Freeform Optics", PhD Thesis, Technische Universiteit Eindhoven, ISBN 978-90-386-1607-0, 2009 